\title{
Minimal residual disease negativity by next- generation flow cytometry is associated with improved organ response in AL amyloidosis
}

Giovanni Palladini $\oplus^{1,2,3}$, Bruno Paiva (1) ${ }^{4}$, Ashutosh Wechalekar5, ${ }^{5,6}$, Margherita Massa ${ }^{2}$, Paolo Milani ${ }^{1,2}$, Marta Lasa ${ }^{4}$, Sriram Ravichandran @ ${ }^{5}$, Isabel Krsnik7, Marco Basset ${ }^{1,2,3}$, Leire Burgos ${ }^{4}$, Mario Nuvolone ${ }^{1,2,3}$, Ramón Lecumberri', Andrea Foli ${ }^{1,2}$, Noemi Puig ${ }^{8}$, Melania Antonietta Sesta ${ }^{1,2,3}$, Margherita Bozzola ${ }^{1,2,3}$, Pasquale Cascino ${ }^{1,2,3}$, Alice Nevone ${ }^{1,2,3}$, Jessica Ripepi ${ }^{1,2,3}$, Pierpaolo Berti $\mathbb{1}^{9}$, Simona Casarini $\mathbb{C B}^{1,2}$, Ombretta Annibali ${ }^{10}$, Alberto Orfao $\mathbb{E}^{8}$, Jesus San-Miguel (10 ${ }^{4}$ and Giampaolo Merlini (iD)

\begin{abstract}
Light chain (AL) amyloidosis is caused by a small B-cell clone producing light chains that form amyloid deposits and cause organ dysfunction. Chemotherapy aims at suppressing the production of the toxic light chain (LC) and restore organ function. However, even complete hematologic response (CR), defined as negative serum and urine immunofixation and normalized free LC ratio, does not always translate into organ response. Next-generation flow (NGF) cytometry is used to detect minimal residual disease (MRD) in multiple myeloma. We evaluated MRD by NGF in $92 \mathrm{AL}$ amyloidosis patients in CR. Fifty-four percent had persistent MRD (median 0.03\% abnormal plasma cells). There were no differences in baseline clinical variables in patients with or without detectable MRD. Undetectable MRD was associated with higher rates of renal ( $90 \%$ vs $62 \%, p=0.006$ ) and cardiac response (95\% vs $75 \%, p=0.023$ ).

Hematologic progression was more frequent in MRD positive ( 0 vs $25 \%$ at 1 year, $p=0.001$ ). Altogether, NGF can detect MRD in approximately half the AL amyloidosis patients in $C R$, and persistent MRD can explain persistent organ dysfunction. Thus, this study supports testing MRD in CR patients, especially if not accompanied by organ response. In case MRD persists, further treatment could be considered, carefully balancing residual organ damage, patient frailty, and possible toxicity.
\end{abstract}

\section{Introduction}

Light chain (AL) amyloidosis is caused by a small B-cell clone, more commonly a plasma cell (PC) clone with shared genetic features with multiple myeloma (MM) and monoclonal gammopathy of undetermined significance, producing light chains (LCs) that form amyloid deposits and exert toxicity on target organs ${ }^{1-3}$.

\footnotetext{
Correspondence: Giovanni Palladini (giovanni.palladini@unipv.it)

${ }^{1}$ Amyloidosis Research and Treatment Center, "Fondazione Istituto di Ricovero

e Cura a Carattere Scientifico (IRCCS) Policlinico San Matteo", Pavia, Italy

'Biochemistry, Biotechnology and Advanced Diagnostics Laboratory,

"Fondazione Istituto di Ricovero e Cura a Carattere Scientifico (IRCCS)

Policlinico San Matteo", Pavia, Italy
}

Full list of author information is available at the end of the article
Lymphoplasmacytic clones sustain the disease in a minority of patients ${ }^{4}$. The size of the underlying PC clone at baseline affects prognosis ${ }^{5}$. Profound decreases of LC levels through PC-targeting chemotherapy can result in the improved organ dysfunction and arrest the otherwise inexorable progression of the disease ${ }^{6-10}$. After treatment, even small increases in amyloid LCs can cause organ progression and reduced survival ${ }^{11}$. Current hematologic and organ response criteria are based on $\mathrm{M}$ protein studies and on changes in the difference between amyloidogenic (involved; iFLC) and uninvolved free LC (dFLC) and in markers of organ dysfunction ${ }^{12,13}$. Amyloid complete response $(\mathrm{aCR})$ is defined by normal FLC ratio plus 
negative serum and urine immunofixation, and predicts prolonged survival ${ }^{12}$. However, even aCR does not translate into organ response in all patients. This could be explained by irreversible organ damage, permanence of amyloid deposits, or persistence of undetectable levels of amyloid LCs produced by treatment-resistant residual PCs, possibly combined.

High-sensitive next-generation flow cytometry (NGF) is used to detect minimal residual disease (MRD) in $\mathrm{MM}^{14}$. As compared to previous, less sensitive flow cytometry methods, NGF offers robust surrogate endpoints for clinical trials and guidance for treatment ${ }^{14-18}$. Accordingly, it has been suggested as the new treatment endpoint for $\mathrm{MM}^{19}$. Evaluation of MRD in AL amyloidosis is an emerging area of interest ${ }^{20-27}$. The group at Mayo Clinic showed that lack of clonal bone marrow (BM) PCs by standard-sensitivity multiparameter flow cytometry, is associated with improved progression-free survival ${ }^{25,26}$. More recently, the Boston group reported trend for higher probability of organ response in patients in $\mathrm{aCR}$ and undetectable $\mathrm{MRD}^{28}$. However, there are no studies evaluating the incremental clinical significance of MRD monitoring by high-sensitivity NGF over routine monoclonal protein studies for hematologic response assessment in patients with AL amyloidosis otherwise in aCR. Thus, it remains unknown if, similarly to $\mathrm{MM}^{16}$, a flow MRD-negative criteria should be adopted in AL amyloidosis.

\section{Methods}

From April 2016 to July 2019, 92 patients confirmed to be in aCR at least 6 months after treatment discontinuation were selected for NGF-based MRD assessment at 14 participating centers in Italy, Spain, and the United Kingdom (Supplemental Table 1). Patients with MM, defined as $>60 \% \mathrm{BMPC}$, and/or involved/uninvolved FLC ratio $>100$, and/or bone lesions, as well as patients with IgM-related AL amyloidosis were excluded. All patients gave written informed consent for their clinical data to be used for research purposes.

Clinical variables were recorded at the time of diagnosis, at the time of first documentation of aCR, at the time of MRD assessment, and at each subsequent evaluation. Clinical decisions (e.g., treatment and frequency of subsequent evaluations) were protocolized and were not influenced by the presence or absence of MRD, except for a single patient with MRD and organ progression who started rescue treatment. All patients who maintained aCR 6 months after the first assessment were asked to perform BM aspiration for MRD evaluation. The patients who no longer satisfied criteria of aCR were excluded from the final analysis.

Hematologic and organ responses and hematologic relapse from aCR were defined based on current criteria $^{12,13,29}$. aCR required both negative serum and urine immunofixation and normal FLC ratio.

Cardiac and renal responses were evaluated at the time of first documentation of aCR (compared to data at diagnosis) and at the time of MRD assessment (compared to data at the time of first documentation of aCR). Organ response was also assessed comparing data obtained at the time of MRD assessment with those obtained at the time of diagnosis. Cardiac response was defined as a decrease both $>30 \%$ and $>300 \mathrm{ng} / \mathrm{L}$ in NT-proBNP. The NT-proBNP level needed to be $>650 \mathrm{ng} / \mathrm{L}$ for cardiac response to be measurable. Renal response was defined as a decrease of at least $30 \%$ or $<0.5 \mathrm{~g} / 24 \mathrm{~h}$ of proteinuria in the absence of a decrease $>25 \%$ in estimated glomerular filtration rate in patients whose proteinuria was at least $0.5 \mathrm{~g} / 24 \mathrm{~h}$.

Hematologic relapse from aCR was defined by the reappearance of a monoclonal component in serum and or urine at immunofixation, and/or by an abnormal FLC ratio.

NGF cytometry on BM aspirates was performed according to the EuroFlow protocol at two different centers (Supplemental Table 1), with harmonized preanalytical and analytical procedures. Samples not processed and analyzed locally were shipped overnight to one of the two evaluating centers (Supplemental Table 1). NGF-based antibody combinations were used for characterizing MRD in BM aspirates (according to EuroFlow) ${ }^{14}$. Briefly, the EuroFlow lyse-wash-and-stain standard sample preparation protocol and the 2-tube 8color EuroFlow NGF antibody panel was performed for identification of BMPC, and discrimination between phenotypically aberrant and normal PC. Tube 1 included CD138-BV421, CD27-BV510, CD38-FITC, CD56-PE, CD45-PerCPCy5.5, CD19-PECy7, CD117-APC, and $\mathrm{CD} 81-\mathrm{APCH} 7$ monoclonal antibodies. In tube 2, the CD117-APC and CD81-APCH7 monoclonal antibodies were replaced by CyIgKappa-APC and CyIgLambdaAPCH7, respectively. Labeled antibodies were purchased from Cytognos S.L. (Salamanca, Spain), BD Biosciences (San Jose, CA, USA), BioLegend Inc. (San Diego, CA, USA), Beckman Coulter (Brea, CA, USA), and DAKO (Glostrup, Denmark). The two-tube strategy allows detection of clonality with specific confirmation of LC restriction on phenotypically aberrant $\mathrm{PC}$, identified by antigen under-expression (CD19, CD27, CD38, CD45, and CD81) or overexpression (CD56, CD117, CD138) as compared to normal PCs. In accordance with the guidelines for MRD response criteria in $\mathrm{MM}^{16}$, a minimum sensitivity of $10^{-5}$ was achieved in all patients and $10^{-6}$ sensitivity was reached in 70/92 (76\%) cases. Data were analyzed using the Infinicyt software (version 1.7; Cytognos Salamanca, Spain) by operators blind to clinical data. The percentage of B-cell precursors, nucleated red 
Table 1 Patients characteristics.

\begin{tabular}{|c|c|c|c|}
\hline Variable & $\begin{array}{l}\text { MRD positive }(N=50) N \\
(\%) / \text { median }(\mathrm{IQR})\end{array}$ & $\begin{array}{l}\text { MRD negative }(N=42) N \\
(\%) / \text { median }(\mathrm{IQR})\end{array}$ & $P$ value \\
\hline Male sex & $33(66)$ & $21(50)$ & 0.128 \\
\hline Age at diagnosis, years & $59(55-66)$ & $61(55-68)$ & 0.280 \\
\hline \multicolumn{4}{|l|}{ Organ involvement at diagnosis } \\
\hline Heart & $36(67)$ & $23(54)$ & 0.093 \\
\hline Kidney & $30(60)$ & $31(73)$ & 0.172 \\
\hline Liver & $11(22)$ & $4(9)$ & 0.117 \\
\hline Cardiac stage at diagnosis & (Available in 45 patients) & (Available in 40 patients) & \\
\hline । & $11(24)$ & $17(43)$ & 0.084 \\
\hline$\|$ & $18(40)$ & $12(30)$ & 0.341 \\
\hline IIla & $15(34)$ & $9(22)$ & 0.227 \\
\hline$\| l l b$ & $1(2)$ & $2(5)$ & 0.582 \\
\hline Renal stage at diagnosis & (Available in 41 patients) & (Available in 39 patients) & \\
\hline I & $20(49)$ & $18(46)$ & 0.818 \\
\hline$\|$ & $18(44)$ & $18(46)$ & 0.843 \\
\hline III & $3(7)$ & $3(8)$ & 0.951 \\
\hline $\mathrm{eGFR}, \mathrm{mL} / \mathrm{min}$ per $1.73 \mathrm{~m}^{2}$ & $86(61-90)$ & $76(60-89)$ & 0.158 \\
\hline BMPC at diagnosis (\%) & $8(4-14)$ & $9(4-15)$ & 0.865 \\
\hline $\mathrm{dFLC}$ at diagnosis, mg/L & $141(65--488)$ & $112(34-397)$ & 0.303 \\
\hline Exposure to two lines of therapy before $a C R$ & $25(50)$ & $15(36)$ & 0.177 \\
\hline $\begin{array}{l}\text { Time from diagnosis to first aCR } \\
\text { documentation, months }\end{array}$ & $10(6-15)$ & $11(5-19)$ & 0.823 \\
\hline $\begin{array}{l}\text { Time from first aCR documentation to MRD } \\
\text { assessment, months }\end{array}$ & $13(5-30)$ & $12(6-37)$ & 0.368 \\
\hline Autologous stem cell transplant & $19(38)$ & $16(38)$ & 0.991 \\
\hline Melphalan & $23(46)$ & $14(33)$ & 0.227 \\
\hline Bortezomib & $45(90)$ & $36(86)$ & 0.547 \\
\hline Cardiac response at the time aCR was documented & $16 / 29(55)$ & 15/21 (71) & 0.262 \\
\hline Renal response at the time aCR was documented & $12 / 29(41)$ & 19/31 (61) & 0.470 \\
\hline
\end{tabular}

Cardiac stage is defined by N-terminal pro-natriuretic peptide type B (NT-proBNP, cutoff $332 \mathrm{ng} / \mathrm{L}$ ) and cTnl (cutoff $0.1 \mathrm{ng} / \mathrm{mL}$ ), with stages I-III patients having none, one, or to markers above the cutoff, respectively. Stage Illa patients have NT-proBNP $<8500 \mathrm{ng} / \mathrm{L}$. Stage IIlb patients have NT-proBNP $>8500 \mathrm{ng} / \mathrm{L}$. Renal stage is defined by eGFR (cutoff $50 \mathrm{~mL} / \mathrm{min}$ per $1.73 \mathrm{~m}^{2}$ ) and proteinuria (cutoff $5 \mathrm{~g} / 24 \mathrm{~h}$ ); stage I patients have both eGFR above and proteinuria below the cutoff, stage II have either eGFR below or proteinuria above the cutoff, and stage III patients have both eGFR below and proteinuria above the cutoff.

eGFR estimated glomerular filtration rate, $d F L C$ difference between involved (amyloidogenic) and uninvolved free light chain, $a C R$ amyloid complete response defined by negative serum and urine immunofixation and normal free light chain ratio, BMPC bone marrow plasma cell infiltrate, MRD minimal residual disease.

blood cells, and mast cells was evaluated in each sample to determine the extent of hemodilution.

Fisher exact test was used to assess differences between subgroups, and long-rank test to compare times to progression.

\section{Results}

A total of 108 patients with AL amyloidosis who were known to be in aCR at least 6 months after treatment discontinuation, underwent NGF-based MRD assessment. Sixteen patients (15\%) did not maintain aCR at the time the BM specimen for MRD assessment was obtained, and were excluded for the study. In these 16 subjects, a median of 447 PCs with abnormal phenotype (range 54-3581), corresponding to $0.02 \%$ (range $0.002-0.335 \%$ ) were detected. The remaining, 92 patients with confirmed $\mathrm{aCR}$ at the time of MRD assessment were considered for the analysis (Table 1). Median time between first 


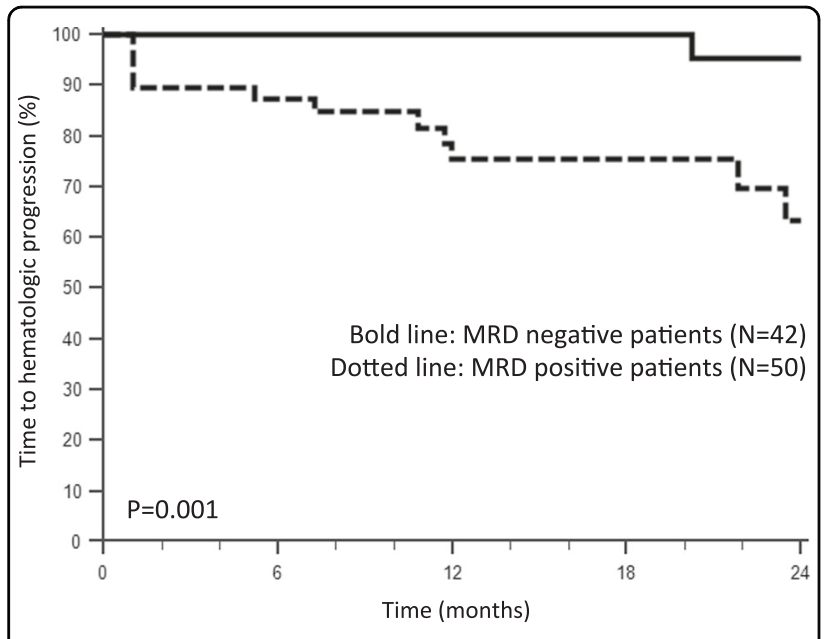

Fig. 1 Maintenance of amyloid complete response after MRD assessment according to MRD status. Bold line: MRD negative patients $(N=42)$. Dotted line: MRD positve patients $(N=50)$.

achievement of aCR and MRD assessment was 11 months (interquartile range 9-30 months). Patients were exposed to one (52 cases, $56 \%$ ) or two ( 40 cases, $44 \%$ ) lines of therapy before aCR was reached and MRD was assessed.

Fifty patients (54\%) had detectable MRD (median $0.02701 \%$, range $0.0002-0.33010 \%$ ). There was no significant difference in clinical variables measured at baseline in patients with and without detectable MRD. Moreover, the number of lines of treatment performed before evaluation, the median time from diagnosis to first $\mathrm{aCR}$ documentation and the median time from first aCR documentation to MRD assessment were not different between MRD positive and negative patients. At the time of MRD assessment, dFLC levels ranged from $0 \mathrm{mg} / \mathrm{L}$ to $29 \mathrm{mg} / \mathrm{L}$ and were $<10 \mathrm{mg} / \mathrm{L}$ in 67 patients $(73 \%)$ in the entire study population. Of note, dFLC levels were significantly lower in patients with undetectable MRD (median 1.5 vs $6.5 \mathrm{mg} / \mathrm{L}, P=0.001$ ).

Patients with undetectable MRD were more likely to attain renal $[92 \%$ (23/25 evaluable) vs $57 \%$ (15/26 evaluable), $P=0.005]$ and cardiac response [95\% (18/19 evaluable) vs $71 \%(20 / 28$ evaluable), $P=0.046]$. Importantly, time between diagnosis and aCR, or between achievement of $\mathrm{aCR}$ and MRD assessment was not associated with organ response (Supplementary Table 2). As above mentioned, organ response was assessed by comparing the organ function at the time of MRD assessment with data obtained at the time of diagnosis. Interestingly, the rate of renal response was higher in the MRD negative cohort: $90 \%$ (28/31 evaluable) vs $62 \%$ ( $18 / 29$ evaluable), $P$ $=0.006]$. The same pattern was observed for cardiac response: $95 \%$ (22/23 evaluable) vs $75 \%$ ( $24 / 32$ evaluable), $P=0.023]$ for patients with undetectable vs detectable MRD, respectively.
After a median follow-up of 23 months from the time of MRD assessment, three patients, all with persistent MRD, died. The difference in overall survival between patients with and without detectable MRD did not reach statistical significance $(P=0.203)$. Time to hematologic progression was significantly longer in MRD-negative patients: only 1 patient with undetectable MRD (sensitivity reached, $10^{-5}$ ) progressed, compared to 13 MRD-positive patients (Fig. 1). Interestingly, rate of hematologic progression at 1 year after MRD assessment was not different in patients who received one or two lines of therapy (10\% vs $15 \%$ progressing at 1 year, $P=0.763$ ) and in patients who received or did not receive autologous stem cell transplant (5\% vs $10 \%$ progressing at 1 year, $P=0.278$ ).

\section{Discussion}

We report here on the largest population of patients with AL amyloidosis with sustained CR undergoing MRD assessment with NGF. With a minimum sensitivity of $10^{-5}$ (reaching $10^{-6}$ in $76 \%$ of cases), persistent MRD was identified in $54 \%$ of cases. This proportion does not appear to be affected by exposure to one vs two lines of therapy, and time to achievement of aCR or to assessment of MRD. This finding is in agreement with the observations by Kastritis et al. who analyzed 20 patients in aCR (40\% after ASCT) and reported MRD negativity in 8 $(40 \%)^{24}$. Similarly, Muchtar et al. analyzed patients at the end of first-line treatment (84\% after ASCT) and reported that, among 16 subjects in aCR, 8 (50\%) had undetectable clonal PCs by multiparameter flow cytometry ${ }^{26}$. In a more recent case series from the Mayo Clinic, where MRD assessment was performed within 2 years from start of therapy (in $57 \%$ of cases after ASCT), MRD negativity was observed in 15 out of 20 (75\%) patients in aCR. In the Boston series, $55 \%$ of patients in CR were MRD positive and a trend to a better organ (especially renal) response was noted in those who reached MRD negativity. While small sample size and differences in patient selection criteria may at least partially account for the increased rate of MRD negativity in this study, the lower sensitivity of MRD assessment $\left(\geq 10^{-5}\right.$, with 11 out of 22 patients in VGPR found to be MRD negative) could also have played a role. Table 2 summarizes the methodology and findings of published flow cytometry-based MRD studies in AL amyloidosis and of the present report. The fact that currently available therapies can yield profound, MRDnegative responses in up to $50 \%$ of patients with $\mathrm{AL}$ amyloidosis in aCR is encouraging and can explain the long-term progression-free survival of patients in aCR. These promising results will probably improve when novel, powerful drugs such as daratumumab, will become accessible.

In the present study, undetectable MRD was associated with a further improvement of organ involvement after 


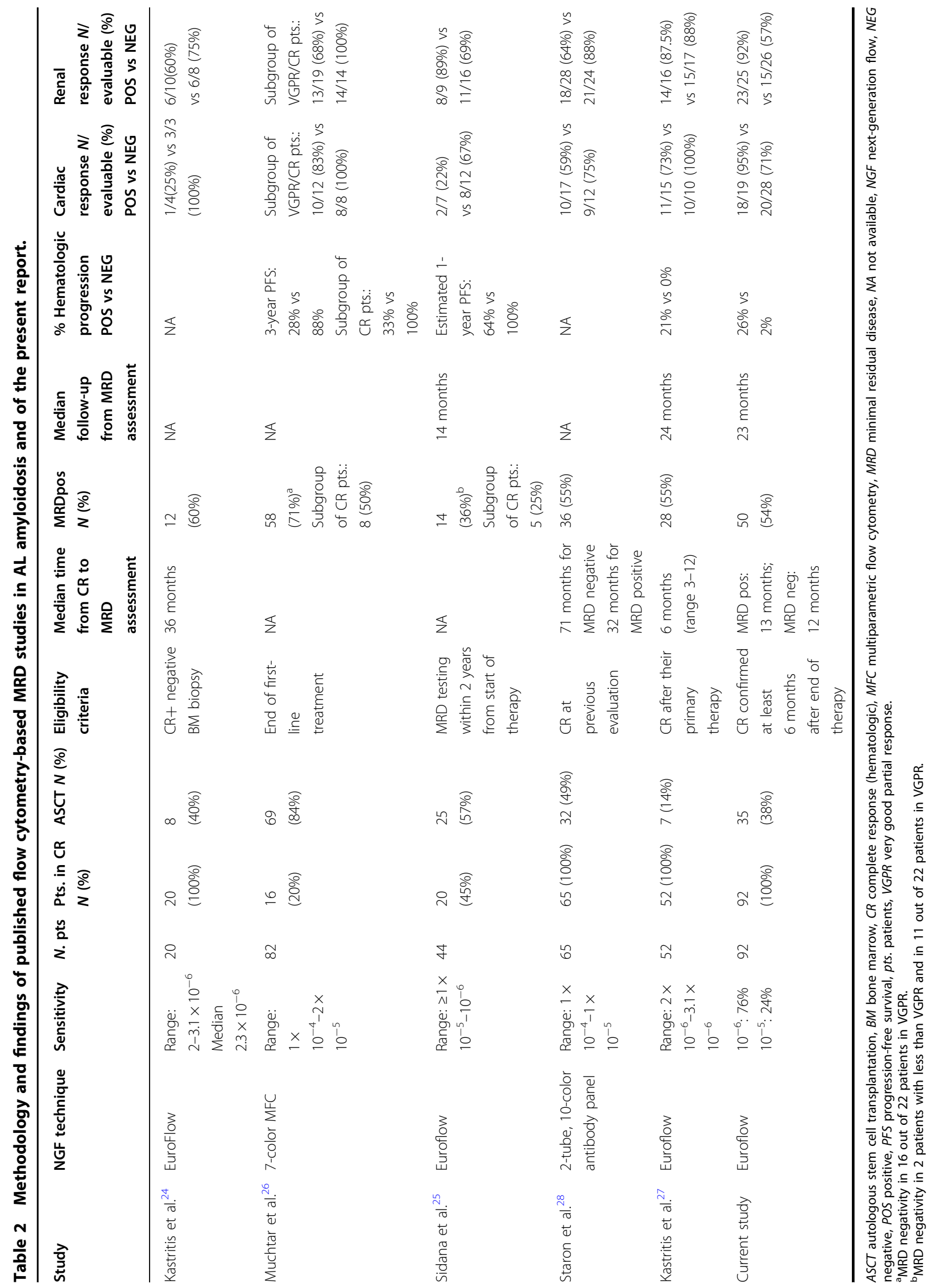


aCR in $>90 \%$ of patients. Both renal and cardiac response rates were higher in the MRD-negative cohort and also from diagnosis to MRD assessment. This links persistence of organ dysfunction and damage with permanence of even minimal clonal disease, producing undetectable, but still toxic amounts of LCs. This observation further corroborates other clinical and laboratory data, indicating a toxic effect of the circulating amyloid precursor ${ }^{30-33}$. Thus, efforts to improve the rate of organ response should aim at deepening hematologic response, possibly eradicating the PC clone. As such, high-sensitive NGF becomes a clinically relevant biomarker to confirm if the achievement of aCR is associated with profound eradication of clonal PCs, and to monitor the reappearance of MRD before hematological relapse as potential surrogate of upcoming organ dysfunction.

Undetectable MRD was also associated with longer progression-free survival. About one quarter of patients with persistent MRD experienced a hematologic progression (loss of aCR), while only one patient progressed among the 42 patients with MRD negativity. Of note, the sensitivity achieved by NGF for this single patient progressing was $10^{-5}$, further stressing the importance of reaching the highest sensitivity threshold with current MRD methodologies (i.e., $2 \times 10^{-6}$ for NGF and $1 \times 10^{-6}$ for NGS).

In conclusion, NGF can detect MRD in patients with $\mathrm{AL}$ amyloidosis otherwise in $\mathrm{aCR}$, and persistent MRD can explain persistent organ dysfunction and predict/anticipate hematologic progression. Testing for MRD should be offered to subjects who attain aCR, especially if aCR is not accompanied by organ response. In case MRD is present, further chemotherapy could be considered, carefully balancing residual organ damage, patient frailty, and possible toxicity.

\section{Acknowledgements}

This study was supported by a grant from CARIPLO "Molecular mechanisms of Ig toxicity in age-related plasma cell dyscrasias no. 2015-0591", by a grant from the Black Swan Research Initiative from the International Myeloma Foundation "Automated multidimensional flow cytometry for high-sensitive screening and to monitor response in AL amyloidosis", by a grant from CARIPLO

"Structure-function relation of amyloid: understanding the molecular bases of protein misfolding diseases to design new treatments no. 2013-0964", by a grant from the Amyloidosis Foundation "Investigating new therapies to treat AL amyloidosis", and by a grant from Cancer Research UK, FCAECC and AIRC under the Accelerator Award 2017 Program "Early detection and intervention: understanding the mechanisms of transformation and hidden resistance of incurable hematological malignancies", by a grant from CARIPLO "Harnessing the plasma cell secretory capacity against systemic light chain amyloidosis" (no. 2018-0257), by a grant from the Italian Ministry of Health "Towards effective, patient-tailored anti-plasma cell therapies in AL amyloidosis: predicting drug response and overcoming drug resistance" (GR-201812368387). This study has also supported the Centro de Investigación Biomédica en Red_Área de Oncología_del Instituto de Salud Carlos III (CIBERONC; CB16/12/00369, CB16/12/00400, and CB16/12/00489) and the Instituto de Salud Carlos III/Subdirección General de Investigación Sanitaria (FIS No. PI13/02196). G.P. is supported in part by the Bart Barlogie Young Investigator Award from the International Myeloma Society (IMS). P.M. is supported in part by a fellowship grant form Collegio Ghislieri (Pavia). We acknowledge the study coordinator and data manager Anna Carnevale Baraglia.

\section{Author contibutions}

G.P. designed the study, evaluated patients, collected data, analyzed data, and wrote the manuscript and gave final approval of the manuscript. P.M. and M. Basset evaluated patients, collected data, analyzed data, critically reviewed the manuscript, and gave final approval of the manuscript. M.N. collected data, wrote the manuscript, critically reviewed the manuscript. and gave final approval of the manuscript. B.P., M.M., M.A.S., L.B., and N.P. performed NGF analysis, collected data, critically reviewed the manuscript, and gave final approval of the manuscript. M.L., M. Bozzola, P.C., A.N., S.C., and A.F. collected data, critically reviewed the manuscript, and gave final approval of the manuscript. P.B., O.A., and I.K. evaluated patients, collected data, critically reviewed the manuscript, and gave final approval of the manuscript. G.M. designed the study, evaluated patients, critically reviewed the manuscript, and gave final approval of the manuscript. B.P., A.O., and J.S.-M. designed the study, critically reviewed the manuscript, and gave final approval of the manuscript.

\section{Author details}

${ }^{1}$ Amyloidosis Research and Treatment Center, "Fondazione Istituto di Ricovero e Cura a Carattere Scientifico (IRCCS) Policlinico San Matteo", Pavia, Italy. ${ }^{2}$ Biochemistry, Biotechnology and Advanced Diagnostics Laboratory, "Fondazione Istituto di Ricovero e Cura a Carattere Scientifico (IRCCS) Policlinico San Matteo", Pavia, Italy. 'Department of Molecular Medicine, University of Pavia, Pavia, Italy. ${ }^{4}$ Clinica Universidad de Navarra, Centro de Investigacion Medica Aplicada (CIMA), IDISNA, CIBERONC CB16/12/00369 Pamplona, Pamplona, Spain. ${ }^{5}$ National Amyloidosis Centre, University College London (Royal Free Campus), London, UK. ${ }^{6}$ Department of Haematology, University College London Hospitals, London, UK. ${ }^{7}$ Hospital Universitario Puerta del Hierro, Madrid, Spain. ${ }^{8}$ Servicio General de Citometría, Universidad de Salamanca, IBSAL, and IBMCC CSIC-USAL, CIBERONC, Salamanca, Spain. ${ }^{9}$ Immunohematology and Transfusion Medicine Unit, Department of Laboratories, IRCCS Bambino Gesù Children's Hospital, Rome, Italy.

${ }^{10}$ Hematology and Stem Cell Transplantation Unit, University Campus Biomedico, Rome, Italy

\section{Conflict of interest}

G.P. reports honoraria for lectures from and membership on advisory boards with Jannsen-Cilag. B.P. reports honoraria for lectures from and membership on advisory boards with Amgen, Bristol-Myers Squibb, Celgene, Janssen-Cilag, Merck, Novartis, Roche, and Sanofi; unrestricted grants from Celgene, EngMab, Sanofi, and Takeda; and consultancy for Celgene, Janssen, and Sanofi; P.M. reports honoraria for lectures from Pfizer, Jannsen-Cilag, and travel support from Celgene; M.N. reports honoraria for lectures from Jannsen-Cilag; and R.L. received lecture fees from Jannsen-Cilag.

\section{Publisher's note}

Springer Nature remains neutral with regard to jurisdictional claims in published maps and institutional affiliations.

Supplementary information The online version contains supplementary material available at https://doi.org/10.1038/s41408-021-00428-0.

Received: 9 June 2020 Revised: 17 October 2020 Accepted: 2 November 2020

Published online: 16 February 2021

\section{References}

1. Merlini, G. et al. Systemic immunoglobulin light chain amyloidosis. Nat. Rev. Dis. Primers 4, 38 (2018).

2. Cohen, O. C. \& Wechalekar, A. D. Systemic amyloidosis: moving into the spotlight. Leukemia 34, 1215-1228 (2020).

3. Chattopadhyay, S. et al. Eight novel loci implicate shared genetic etiology in multiple myeloma, AL amyloidosis, and monoclonal gammopathy of unknown significance. Leukemia 34, 1187-1191 (2020). 
4. Sidana, S. et al. IgM AL amyloidosis: delineating disease biology and outcomes with clinical, genomic and bone marrow morphological features. Leukemia 34, 1373-1382 (2020).

5. Muchtar, E. et al. Bone marrow plasma cells $20 \%$ or greater discriminate presentation, response, and survival in AL amyloidosis. Leukemia 34, 1135-1143 (2020)

6. Sidana, $\mathrm{S}$. et al. Revisiting complete response in light chain amyloidosis. Leukemia 34, 1472-1475 (2020).

7. Manwani, R. et al. A prospective observational study of 915 patients with systemic AL amyloidosis treated with upfront bortezomib. Blood. 134 2271-2280 (2019).

8. Milani, $\mathrm{P}$. et al. Indicators of profound hematologic response in $\mathrm{AL}$ amyloidosis: complete response remains the goal of therapy. Blood. Cancer J. 10, 90 (2020).

9. Sidana, S. et al. A validated composite organ and hematologic response model for early assessment of treatment outcomes in light chain amyloidosis. Blood Cancer J. 10, 41 (2020)

10. Sarosiek, S. et al. Comparing measures of hematologic response after highdose melphalan and stem cell transplantation in AL amyloidosis. Blood Cancer J 10, 88 (2020).

11. Palladini, G. et al. Presentation and outcome with second-line treatment in AL amyloidosis previously sensitive to nontransplant therapies. Blood $\mathbf{1 3 1}$ 525-532 (2018)

12. Palladini, G. et al. New criteria for response to treatment in immunoglobulin light chain amyloidosis based on free light chain measurement and cardiac biomarkers: impact on survival outcomes. J. Clin. Oncol. 30, 4541-4549 (2012).

13. Palladini, G. et al. A staging system for renal outcome and early markers of renal response to chemotherapy in AL amyloidosis. Blood 124, 2325-2332 (2014).

14. Flores-Montero, J. et al. Next generation flow for highly sensitive and standardized detection of minimal residual disease in multiple myeloma. Leukemia 31, 2094-2103 (2017)

15. Paiva, B., van Dongen, J. J. \& Orfao, A. New criteria for response assessment: role of minimal residual disease in multiple myeloma. Blood $\mathbf{1 2 5}$ 3059-3068 (2015).

16. Kumar, S. et al. International Myeloma Working Group consensus criteria for response and minimal residual disease assessment in multiple myeloma. Lancet Oncol. 17, e328-e346 (2016).

17. Paiva, B. et al. Measurable residual disease by next-generation flow cytometry in multiple myeloma. J. Clin. Oncol. 38, 784-792 (2019).

18. Facon, T. et al. Carfilzomib or bortezomib with melphalan-prednisone for transplant-ineligible patients with newly diagnosed multiple myeloma. Blood. 133, 1953-1963 (2019).
19. Harousseau, J. L. \& Avet-Loiseau, H. Minimal residual disease negativity is a new end point of myeloma therapy. J Clin. Oncol. 35, 2863-2865 (2017)

20. Milani, P., Merlini, G. \& Palladini, G. What does minimal residual disease mean in AL amyloidosis? Expert Opin. Orphan Drugs. 6, 703-705 (2018).

21. Paiva, B. et al. The clinical utility and prognostic value of multiparameter flow cytometry immunophenotyping in light-chain amyloidosis. Blood. 117, 3613-3616 (2011).

22. Lisenko, K. et al. Flow cytometry-based characterization of underlying clonal B and plasma cells in patients with light chain amyloidosis. Cancer Med. $\mathbf{5}$ 1464-1472 (2016)

23. Muchtar, E. et al. The prognostic value of multiparametric flow cytometry in $\mathrm{AL}$ amyloidosis at diagnosis and at the end of first-line treatment. Blood 129 82-87 (2017).

24. Kastritis, E. et al. Evaluation of minimal residual disease using nextgeneration flow cytometry in patients with AL amyloidosis. Blood Cancer J 8, 46 (2018).

25. Sidana, S. et al. Impact of minimal residual negativity using next generation flow cytometry on outcomes in light chain amyloidosis. Am. J. Hematol. 95, 497-502 (2020).

26. Muchtar, E. et al. Survival impact of achieving minimal residual negativity by multi-parametric flow cytometry in AL amyloidosis. Amyloid 27, 13-16 (2020).

27. Kastritis, E. et al. Next generation flow cytometry for MRD detection in patients with AL amyloidosis. Amyloid 1-5 (2020).

28. Staron, A. et al. Assessment of minimal residual disease using multiparametric flow cytometry in patients with AL amyloidosis. Blood Adv. $\mathbf{4}$ 880-884 (2020).

29. Gertz, M. et al. Definition of organ involvement and treatment response in immunoglobulin light chain amyloidosis (AL): a consensus opinion from the 10th International Symposium on Amyloid and Amyloidosis, Tours, France, 1822 April 2004. Am. J. Hematol. 79, 319-328 (2005).

30. Diomede, L. et al. A Caenorhabditis elegans-based assay recognizes immunoglobulin light chains causing heart amyloidosis. Blood. 123, 3543-3552 (2014).

31. Diomede, $L$ et al. Cardiac light chain amyloidosis: the role of metal ions in oxidative stress and mitochondrial damage. Antioxid. Redox Signal. 27, 567-582 (2017)

32. Palladini, G. et al. Circulating amyloidogenic free light chains and serum Nterminal natriuretic peptide type B decrease simultaneously in association with improvement of survival in AL. Blood 107, 3854-3858 (2006)

33. Abeykoon, J. P. et al. Daratumumab-based therapy in patients with heavilypretreated AL amyloidosis. Leukemia 33, 531-536 (2018). 